\title{
Lymphatic Mapping Techniques and Sentinel Lymph Node Biopsy in Breast Cancer
}

\author{
Erika A. Newman, MD ${ }^{\mathrm{a}}$, \\ Lisa A. Newman, MD, MPH, FACS ${ }^{\mathrm{b}, *}$ \\ ${ }^{a}$ Department of Surgery, University of Michigan, 1500 East Medical Center Drive, \\ Ann Arbor, MI 48109-0932, USA \\ ${ }^{\mathrm{b}}$ Breast Center, University of Michigan Comprehensive Cancer Center, 1500 East Medical \\ Center Drive, Ann Arbor, MI 48109-0932, USA
}

The axillary nodal status is accepted universally as the most powerful prognostic tool available for early stage breast cancer. Breast cancer patients routinely undergo surgical staging of the axilla because other primary tumor features are inadequate in predicting the presence versus absence of nodal positivity [1-3]. The status of the axillary lymph nodes also guides treatment options and adjuvant therapies. The removal of level I and level II lymph nodes at axillary node dissection (ALND) is the most accurate method to assess nodal status, and it is the universal standard. ALND is associated with several adverse long-term sequelae including lymphedema, the disruption of nerves in the axilla, chronic shoulder pain, weakness, and joint dysfunction. Additionally, the survival advantage of ALND has been challenged, and less morbid methods of evaluating the axillary nodal basin have been sought.

Breast cancer spreads from the tumor bed to one or a few lymph nodes before it spreads to other axillary nodes. These sentinel nodes can be identified and surgically excised for histological analysis. Lymphatic mapping with sentinel lymph node biopsy (SLNB) has emerged as an effective method of detecting axillary metastases. Veronesi and colleagues [4] randomly assigned 516 women with early stage breast cancer to either SLNB and ALND or SLNB alone (ALND was performed only for axillary metastases in the SLNB-alone arm). The authors demonstrated that SLNB was

* Corresponding author.

E-mail address: lanewman@umich.edu (L.A. Newman). 
accurate and reliable with a false-negative rate of $8 \%$. There was less pain and better arm mobility in those who underwent SLNB only. Additionally, there were no differences in local recurrence or survival at follow-up. The NSABP-32 trial is the largest multicenter trial to date examining the safety and accuracy of SLNB [5]. The trial randomly assigned women with clinically negative axillae to receive SLNB with an ALND or SLNB alone. Early results have demonstrated that SLNB is safe and reliable, with false-negative rates of $8 \%$ to $10 \%$, and lower morbidity than ALND. Although the longterm results are forthcoming, the clinical advantages of SLNB are apparent, and the procedure is becoming the preferred standard by patients and breast cancer surgeons.

Given the rapid growth of lymphatic mapping and SLNB, surgical groups have developed several variations in practice, and many technical aspects of the procedure are evolving. These variations have included the choice of mapping label, radioisotope quantity and processing, label injection site, timing of radioisotope injection, and the use of preoperative lymphoscintigraphy scanning. Because these controversies have not been studied extensively in clinical trials, the method of lymphatic mapping ultimately should be selected based on those methods that have been proven safe, and on the services and resources of a given breast care program. Table 1 summarizes the results of various studies that have analyzed SLNB accuracy as a function of mapping technique [6-18].

\section{Choice mapping label}

\section{Radioisotope alone}

Krag and colleagues [19] first described the use of radioisotope alone for breast cancer in 1993, using technetium-99m sulfur colloid and a hand-held gamma probe. The sentinel node identification rate was $98 \%$, with a falsenegative rate of $11 \%$. Technetium-99m sulfur colloid is the most widely used radioisotope for lymphatic mapping in the United States. In Europe, technetium 99m-colloidal albumin is used most. The specific radioisotope selected for the mapping process is determined largely by availability and by the center's nuclear medicine practices [20]. The doses of radioactive technetium vary by institution and range from 0.1 to $4 \mathrm{mCi}$.

\section{Blue dye}

Isosulfan blue dye (Lymphazurin 1\%, US Surgical Corp, Norwalk, CT) initially was studied extensively in lymphatic mapping for melanoma. The use of isosulfan blue dye as a single agent in SLNB for breast cancer initially was reported by Giuliano and colleagues [6], with sentinel node identification rates of $98 \%$, without false-negative nodes. The major disadvantage of isosulfan blue dye is the risk of life-threatening allergic and anaphylactic 
Table 1

Selected studies evaluating accuracy of sentinel lymph node biopsy as a function of lymphatic mapping technique

\begin{tabular}{|c|c|c|c|c|c|c|c|c|c|c|c|c|c|c|c|}
\hline \multirow[b]{2}{*}{ Study } & \multirow[b]{2}{*}{$\begin{array}{l}\text { Total } \\
\# \\
\text { cases }\end{array}$} & \multirow[b]{2}{*}{$\begin{array}{l}\text { Sentinel } \\
\text { lymph } \\
\text { node (SLN) } \\
\text { identification } \\
\text { rate }(\%)\end{array}$} & \multirow[b]{2}{*}{$\begin{array}{l}\text { SLN } \\
\text { FN } \\
\text { rate } \\
(\%)\end{array}$} & \multicolumn{6}{|c|}{ Factors associated with SLN nonidentification } & \multicolumn{6}{|c|}{ Factors associated with SLN FN risk } \\
\hline & & & & $\begin{array}{l}\text { Learning } \\
\text { curve }\end{array}$ & $\begin{array}{l}\text { Tumor } \\
\text { location } \\
\text { (medial } \\
\text { worse) }\end{array}$ & $\begin{array}{l}\text { Older- } \\
\text { age } \\
\text { patient }\end{array}$ & $\begin{array}{l}\text { Prior } \\
\text { excisional } \\
\text { biopsy }\end{array}$ & $\begin{array}{l}\text { Single } \\
\text { versus dual } \\
\text { mapping } \\
\text { agent }\end{array}$ & $\begin{array}{l}\text { Larger- } \\
\text { sized } \\
\text { tumor }\end{array}$ & $\begin{array}{l}\text { Learning } \\
\text { curve }\end{array}$ & $\begin{array}{l}\text { Tumor } \\
\text { location } \\
\text { (upper outer } \\
\text { quadrant } \\
\text { worse) }\end{array}$ & $\begin{array}{l}\text { Older- } \\
\text { aged } \\
\text { patient }\end{array}$ & $\begin{array}{l}\text { Prior } \\
\text { excisional } \\
\text { biopsy }\end{array}$ & $\begin{array}{l}\text { Single } \\
\text { versus dual } \\
\text { mapping } \\
\text { agent }\end{array}$ & $\begin{array}{l}\text { Larger- } \\
\text { sized } \\
\text { tumor }\end{array}$ \\
\hline Canavese [9] & 212 & $97.1 \%$ & $6.5 \%$ & No & NR & NR & NR & Yes & No & No & NR & NR & NR & No & Yes \\
\hline Albertini [7] & 62 & $92 \%$ & $0 \%$ & NR & NR & NR & $\mathrm{NA}^{\mathrm{a}}$ & Yes & NR & No & No & No & No & No & No \\
\hline $\begin{array}{l}\text { McMasters } \\
{[10]}\end{array}$ & 806 & $88 \%$ & $7.2 \%$ & No & No & Yes & No & Yes & No & No & Yes & No & No & Yes & No \\
\hline Veronesi [8] & 163 & $98 \%$ & $4.7 \%$ & No & No & No & No & $\begin{array}{l}\text { NA ( Tc } \\
\quad \text { only used) }\end{array}$ & Yes & No & Yes & No & No & NA & Yes \\
\hline Veronesi [11] & 376 & $98.7 \%$ & $6.7 \%$ & No & No & NR & NR & No & No & No & No & No & NR & No & No \\
\hline $\operatorname{Cox}[12]$ & 465 & $94.4 \%$ & UK & Yes & NR & NR & No & Yes & NR & Yes & NR & NR & NR & NR & NR \\
\hline Giuliano [6] & 174 & $65.5 \%$ & $8.1 \%$ & Yes & NR & NR & NR & $\begin{array}{l}\text { NA (dye } \\
\text { only used) }\end{array}$ & NR & Yes & NR & NR & NR & NR & NR \\
\hline Bedrosian [13] & $104^{\mathrm{b}}$ & $99 \%$ & $3.3 \%$ & NR & NR & NR & NR & NR & No & NR & NR & NR & NR & NR & No \\
\hline Haigh [14] & $284^{\mathrm{c}}$ & $81.0 \%$ & $3.2 \%$ & NR & No & NR & No & NR & No & Yes & No & NR & No & NR & No \\
\hline Wong [15] & 2206 & $92.5 \%$ & $8.0 \%$ & NR & No & NR & No & NR & Yes & NR & No & NR & No & NR & No \\
\hline Krag [16] & 443 & $93 \%$ & $12.8 \%$ & NR & Yes & Yes & Yes & $\begin{array}{l}\text { NA } \\
\text { (isotope- } \\
\text { only used) }\end{array}$ & No & NR & Yes & No & No & $\begin{array}{l}\text { NA } \\
\text { (isotope- } \\
\text { only used) }\end{array}$ & No \\
\hline O’Hea [17] & 59 & $93 \%$ & $15 \%$ & NR & No & NR & No & Yes & No & Yes & No & NR & No & No & Yes \\
\hline Guenther [18] & 260 & $81.9 \%$ & $\mathrm{NR}^{\mathrm{d}}$ & Yes & Yes & NR & No & $\begin{array}{l}\text { NA (dye only } \\
\text { used) }\end{array}$ & $\mathrm{NA}^{\mathrm{e}}$ & $\mathrm{NA}^{\mathrm{e}}$ & $\mathrm{NA}^{\mathrm{d}}$ & $\mathrm{NA}^{\mathrm{e}}$ & $\mathrm{NA}^{\mathrm{e}}$ & $\mathrm{NA}^{\mathrm{e}}$ & $\mathrm{NA}^{\mathrm{e}}$ \\
\hline
\end{tabular}

Abbreviations: NR, not reported; NA, not applicable.

${ }^{\text {a }}$ Patients with prior excisional biopsy excluded from study.

${ }^{\mathrm{b}}$ All T2 and T3 tumors.

${ }^{\mathrm{c}}$ Including 181 lymphatic mapping cases with prior excisional biopsy.

${ }^{\mathrm{d}}$ Medial location worse.

e Analyses limited to 47 patients with unsuccessful mapping procedures. 
reactions. The reported allergic reaction rate ranges from $1 \%$ to $3 \%[21,22]$. Most reactions consist of urticaria, rash, blue hives, and pruritus [23]. Although rare, anaphylaxis and hypotension also have been reported. Overall, isosulfan blue dye has excellent results for lymphatic mapping in breast cancer, and is the blue dye most commonly used.

Methylene blue also has been successful in SLNB for breast cancer. Simmons and colleagues [24,25] identified the sentinel node in approximately $93 \%$ of patients studied in a cohort of more than 100 patients; concordance with radioisotope was observed in $95 \%$.

Additionally, methylene blue was compared with Isosulfan blue dye by Blessing and colleagues [26] in 2002. The authors found that all patients had high concomitant isotope mapping and similar sentinel node identification rates. Methylene blue is preferred by some authors because of its lower costs, and also because of its lower risk of allergic reactions. Methylene blue must be injected in the subcutaneous tissues; inadvertent injection into the dermis has resulted in severe skin reactions including necrosis and dermolysis.

\section{Combination of blue dye and radioisotope}

Several authors have demonstrated that the combination of radioisotope and blue dye for lymphatic mapping improves the sentinel lymph node (SLN) identification rate. Albertini and colleagues [7] first reported the successful use of lymphatic mapping with both blue dye and radioisotopes prospectively. The results have been confirmed with several studies demonstrating that the combination method improves the sentinel node identification rate, and dual-agent lymphatic mapping has been accepted universally [27]. Some centers have elected to rely on radioisotope mapping alone, given the potentially life-threatening allergic reactions of isosulfan blue dye.

\section{Filtered versus unfiltered radioisotope}

Identification of a radioactive lymph node depends upon adequate uptake of the radioisotope from the breast parenchyma by intramammary lymphatics. The radioisotope must travel from the breast to the sentinel node in a timely fashion. Radioisotope uptake and travel times ultimately depend on the size of the labeled carrier and on the amount of carrier fluid used. Large particles may not migrate to regional nodes at all (those greater than $400 \mathrm{~nm}$ ), and those too small may migrate too quickly to the entire nodal basin, making identification of single sentinel nodes difficult. The size of technitium-99 sulfur colloid may be altered by the selective use of filters and the pore size of filters used. Filtration through 100 or $220 \mathrm{~nm}$ filters has been studied, with goals of particle sizes ranging from 50 to $200 \mathrm{~nm}$. Filtered preparations resulting in smaller particles travel more quickly and may potentially reach more SLNs, including the higher echelon nodes, if there is 
a prolonged interval between injection of radioisotope and surgery [19]. The unfiltered colloid may be less likely to travel to higher echelon nodes, given its larger size and slower transit time. Linehan and colleagues [28] compared the success of SLNB using filtered versus unfiltered technetium-99m sulfur colloid combined with blue dye mapping. Although the authors found no difference in the overall SLN identification rate, there were significantly more SLNs that were radioactive in the unfiltered group versus the filtered group ( $88 \%$ versus $73 \% ; P=.03)$. These results suggest that filtered smaller particles may pass too quickly from the injection site through the nodal basin before the sentinel nodes are removed.

There has been no consensus on the use of filtered versus unfiltered radioisotopes for lymphatic mapping in breast cancer. The various features may be considered advantages or disadvantages, and selection depends upon the timing of surgery in relation to injection times.

\section{Injection site for mapping agents}

\section{Peri-tumoral injection}

In efforts to replicate the intramammary lymphatic pathways that may have been traversed by metastases, the initial data regarding SLNB used peri-tumoral injections of the mapping agents $[5,6]$. For patients who have nonpalpable tumors, this method has proven difficult and time-consuming, because it requires the use of additional imaging modalities to guide the peri-tumoral injection of radioisotopes. Peri-tumoral injections also have a higher potential for shinethrough, where residual radioactivity from the peri-tumoral injection site creates misleading background activity detected by the gamma probe from the axilla. It is for these reasons that alternate injection sites have been pursued.

\section{Subareolar and dermal injection}

Mammary lymphatics develop as radial extensions from the nipple breast bud. Nearly all breast tissue lymphatic drainage passes through the subareolar plexus of Sappey and then into the axillary nodal basin; hence dermal and subareolar injections are potential approaches for the injection of mapping agents. The sites are particularly advantageous for patients who have nonpalpable or multicentric tumors; they also eliminate the shinethrough effect.

A potential disadvantage to subareolar and dermal injections is that up to $10 \%$ of breast cancers may demonstrate nonaxillary lymphatic drainage with sentinel nodes found in the internal mammary or supraclavicular nodal basins; hence not all breast tumors will have the same drainage patterns as the overlying skin and nipple areas. Additionally, subareolar and dermal injection of blue dye may cause considerable postoperative discoloration of the breast (blue breast), which may last for several months. 
Veronesi and colleagues [8] first described subdermal injections of technetium-99m labeled albumin into the dermis overlying the tumor of 163 patients undergoing SLNB and ALND. The authors found that the SLN identification rate was $98 \%$, with a false-negative rate of $4.7 \%$. Several authors have confirmed the reliability of dermal injections by direct comparisons between peri-tumoral and skin radioisotope injections. Borgstein and colleagues [29] studied 33 breast cancer patients undergoing lymphatic mapping, consisting of dermal injections of blue dye and peri-tumoral injections of radioisotope. The authors found $100 \%$ concordance between blue and radioactive SLNs in 30 of the 33 patients studied, without any false negatives. Linehan and colleagues studied 200 patients undergoing SLNB with peritumoral or excisional biopsy site blue dye injections. In the study, half of the patients also received Tc99-sulfur colloid injected peri-tumorally, and the other half received radioisotopes by means of dermal injections. The SLN identification rates were $92 \%$ for those patients receiving intraparenchymal injections of both blue dye and radioisotopes. For those patients receiving intraparenchymal injection of blue dye and dermal injection of radioisotopes, the SLN identification rate was $100 \%$. In both subsets of patients, the concordance between blue-stained and radioactive sentinel nodes was also high ( $97 \%$ and $95 \%$, respectively). Those patients receiving dermal injections of radioisotopes had a greater proportion of sentinel nodes radioactive when compared with the group receiving peri-tumoral injections of radioisotopes $(97 \%$ versus $78 \% ; P<.001)$. A subsequent report compared 134 patients receiving intraparenchymal lymphatic mapping with 164 patients with mapping using intraparenchymal blue dye and dermal injection of radioisotopes [30]. The SLN identification rate was significantly higher in the group receiving dermal injections of radioisotopes ( $98 \%$ versus $89 \%$ ). There was no difference in the false-negative rates $(4.4 \%$ and $4.8 \%)$.

Several authors have studied the differences between lymphatic drainage pathways of intradermal versus intraparenchymal injections of radioisotopes. Shen and colleagues [31] studied the preoperative lymphoscintigraphy scans of 30 patients undergoing lymphatic mapping for cutaneous breast melanomas and 97 patients undergoing lymphatic mapping with peri-tumoral injection for breast cancer. The authors found that there were a higher percentage of nonaxillary SLNs in the melanoma/dermal injection group (26\% versus $5 \%$ ). In the melanoma cases, there were bilateral axillary and supraclavicular drainage sites detected, whereas the breast cancer cases mapped to the ipsilateral axillae. The results demonstrated that axillary drainage patterns varied between peri-tumoral and dermal lymphatics. Additionally, given the importance of the ipsilateral axillary, mammary, and supraclavicular nodal basins in the staging of breast cancer, if drainage to these sites can be detected with dermal injection, the dermal route may be adequate for the staging of breast cancer.

Klimberg and colleagues [32] compared lymphatic mapping using subareolar injections of radioisotope to peri-tumorally injected blue dye. The 
authors found successful mapping in 64 of 68 patients studied (94\%). The SLN identification rate for blue dye was $89.9 \%$ versus an identification rate of $94.2 \%$ for radioisotope. In the study, all blue nodes were also radioactive, indicating that subareolar injection did not miss any axillary SLNs using this method of mapping.

Subareolar and dermal injection sites also have been examined using various areas of the breast for injection. Beitsch and colleagues [33] studied subareolar radioisotope injected into the mirror-image quadrant of the nipple-areolar tissue and peri-tumoral blue dye injections. The SLN identification for blue dye and radioisotopes were $94 \%$ and $99 \%$, respectively, and $99 \%$ of the blue SLNs were also radioactive. Kern [34,35] reported successful results of lymphatic mapping using subareolar injections of blue dye and radioisotopes at the upper, outer aspect of the nipple-areolar tissue.

Although SLNB has proven reliable in women who have unifocal disease, the studies examining the ideal injection site have set the stage for the consideration of SLNB in multicentric and multifocal disease. Tousimis and colleagues [36] reported results from the largest series examining lymphatic mapping in multicentric and multifocal breast cancer. The authors examined 70 patients who underwent mapping using a combination of radioisotopes and blue dye. In the study, 63 patients received a single intradermal injection of radioisotopes directly over the largest tumor, and five patients received radioisotope peri-tumoral radioisotope injections. Additionally, 67 patients received a single intraparenchymal blue dye injection adjacent to the supero-lateral side of the largest invasive tumor or biopsy cavity. The authors found that the accuracy (SLN identification rate of 96\%) and falsenegative rate $(8 \%)$ of SLNB in patients who had multicentric and multifocal breast cancers were comparable to those with unifocal tumors. Though confirmatory studies are warranted, these results demonstrate the feasibility of SLNB in patients who have multicentric and multifocal breast cancer.

\section{Preoperative lymphoscintigraphy}

Patients undergoing lymphatic mapping with radioisotopes most often receive a preoperative lymphoscintigram (PL) to aid in SLN identification. PL typically consists of anterior and lateral views and specific patient positioning to optimize transit time and radioisotope drainage [37]. Scanning routinely is initiated 20 minutes after radioisotope injection, and images are repeated until the primary SLN basin is identified and there is adequate uptake. The patient then is taken to the operating room for SLNB.

It is controversial whether preoperative scanning is of diagnostic value. Many authors have examined the accuracy of the PL, and given the additional time and cost, question its value in improving the identification of sentinel lymph nodes. Proponents of the technique have argued that the scan may guide the timing of surgery when radioisotope injection is 
performed on the same day as the operation. Additionally, PL will identify the primary drainage pattern, and also internal mammary (IM) sentinel nodes. There is no consensus regarding the management IM SLNs that have been identified by PL, and current recommendations for adjuvant therapies have been defined mostly by axillary nodal metastases. McMasters and colleagues [38] evaluated the role of PL in breast cancer. In the study, a PL was performed in 348 of 588 patients $(59 \%)$, and 240 patients did not receive scans. The SLN was identified in 221 of the $240(92 \%)$ patients who did not undergo preoperative scanning. In these patients, the false-negative rate was $1.6 \%$. In those patients receiving a preoperative lymphoscintigram, the SLN was identified in 310 of the $348(89.1 \%)$ patients, with a false-negative rate of $8.7 \%$. The authors found no significant difference in the SLN identification rate, false-negative rate, or number of SLNs removed between patients receiving PL and those proceeding to operation without scanning. Borgstein and colleagues [39] also studied the role of PL in breast cancer patients. The authors found that the intraoperative gamma probe was more sensitive in detecting radioactive nodes in the axilla than the PL, even when delayed images were obtained. In the study, axillary accumulation was absent in 14 of 130 patients receiving PL. The intraoperative gamma probe was unsuccessful in detecting radioactivity in 8 of 130 cases (seven of these patients also had negative PL).

Data have continued to emerge questioning the ability of PL to improve the accuracy of SLNB, and some centers have abandoned the technique, focusing only on resecting SLNs in the axilla, and relying on the intraoperative gamma probe to detect radioactive SLNs. Until there are definitive data regarding the treatment and importance of nonaxillary drainage, the decision to use PL is ultimately the decision of the surgeon and the multidisciplinary breast team.

\section{Timing of radioisotope injection}

Lymphatic mapping with radioisotopes is performed either as a 1- or 2day procedure. The half-life of technitium-99 is approximately 6 hours and must be taken into account when planning SLNB.

The single-day procedure requires breast injection on the morning of surgery, followed by serial imaging at 1 to several hours after injection until the SLN is identified. In some cases, the process can take several hours and may significantly delay the operation. The effect of delay on patients and on operating room scheduling has led some centers to use a 2-day mapping procedure, with injection of radioisotopes 1 day before operation. The 2-day procedure has been criticized because of the concern that it may require higher doses of radioisotopes, or that with prolonged exposure, radioisotopes may move into higher-echelon nonsentinel lymph nodes. Winchester and colleagues [40] evaluated lymphatic mapping with radioisotope injection 
1 day before operation. The study consisted of 180 patients receiving lymphatic mapping and SLNB, with technitium-99 sulfur colloid injected 1 day preoperatively. The authors found that the SLN identification rate was $90 \%$, and was influenced largely by the surgeon's learning curve. Additionally, mapping was improved when $1.0 \mathrm{mCi}$-filtered radioisotope was used (versus 0.5 to $1.0 \mathrm{mCi}$ unfiltered radioisotope). McCarter and colleagues [41] also had successful outcomes using the 2-day procedure. The authors studied 933 patients who received $0.1 \mathrm{mCi}$ of dermal technitium-99 sulfur colloid in $0.05 \mathrm{cc}$ normal saline on the day of surgery, and 387 patients who received $0.5 \mathrm{mCi}$ technitium-99 sulfur colloid dermal injections on the day before operation. All of the patients had peri-tumoral blue dye injections intraoperatively. The median number of SLNs identified in the 2-day group was slightly higher than in the 1-day group, and the mean level of isotope counts was similar between the two groups. Likewise, Solorzano and colleagues [42] reported success with the 2-day lymphatic mapping technique. The authors found that injection of $2.5 \mathrm{mCi}$ technetium sulfur colloid (filtered) peri-tumorally on the day before surgery with lymphoscintigraphy to track drainage resulted in an overall SLN identification of 97.5\%. All positive SLNs with blue dye staining were also radioactive. Based on the current literature, a 2-day lymphatic mapping procedure is safe and reliable for SLNB in breast cancer.

\section{The future and controversies}

In addition to those previously mentioned controversial areas, the prognostic value of axillary nodal micrometastases identified by immunohistochemistry (IHC) analysis for cytokeratin is unknown, although the topic is the subject of ongoing clinical trials. Because it is not proven that micrometastases have any effect on breast cancer treatment, recurrences, or survival, IHC is generally not included as a routine component of SLNB [43].

Patients who have negative sentinel lymph nodes are safely spared an ALND. The question remains if patients who have positive SLNs gain a survival advantage from completion node dissection. The American College of Surgeons Oncology Group sought to answer this question with a prospective randomized controlled trial of ALND in women with early stage breast cancer and a positive SLNB [44]. The trial was terminated early because of poor patient accrual. Until data from clinical trials are available, completion of ALND remains the standard treatment for patients who have positive SLNs.

Finally, although SLNB is becoming standard for early stage breast cancer, the role of SLNB in patients who have locally advanced disease, and those receiving neoadjuvant chemotherapy (NCTX) is not well established. Breast cancer patients receiving neoadjuvant chemotherapy may undergo pre-chemotherapy sentinel lymph node biopsy (as a definitive staging 
procedure at presentation) or post-chemotherapy (to document the posttreatment nodal status). The pre-chemotherapy strategy commits many patients to a completion ALND on the basis of pre-treatment nodal positivity, thereby negating some of the neoadjuvant chemotherapy downstaging benefits. Several groups have studied the performance of SLNB after NCTX [45-47]. This sequence has been criticized for wide variations in the falsenegative rates. The optimal strategy for incorporating lymphatic mapping into neoadjuvant chemotherapy regimens has yet to be determined and is the subject of ongoing research.

\section{Summary}

The value of SLNB in the staging and prognosis of breast cancer patients with early stage disease is defined clearly, and lymphatic mapping is becoming the standard of care for most centers. It is projected that SLNB will soon replace ALND completely as the initial evaluation procedure of the axillary nodal basin for metastases. As the specifics of lymphatic mapping evolve, the process should be individualized and tailored to institutional capabilities and the practice preferences of the entire multidisciplinary team to yield the most consistent and reliable results.

\section{References}

[1] Carter CL, Allen C, Henson DE. Relation of tumor size, lymph node status, and survival in 24,740 breast cancer cases. Cancer 1989;63:181-7.

[2] Rivadeneira DE, Simmons RM, Christos PJ, et al. Predictive factors associated with axillary lymph node metastases in T1a and T1b breast carcinomas: analysis in more than 900 patients. J Am Coll Surg 2000;191:1-8.

[3] Gann PH, Colilla SA, Gapstur SM, et al. Factors associated with axillary lymph node metastasis from breast carcinoma: descriptive and predictive analyses. Cancer 1999;86: $1511-9$.

[4] Veronesi U, Paganelli G, Giuseppe V, et al. A randomized comparison of sentinel node biopsy with routine axillary dissection in breast cancer. N Engl J Med 2003;349:546-53.

[5] Krag DN, Julian TB, Harlow SP, et al. NSABP-32: phase III, randomized trial comparing axillary resection with sentinel lymph node resection: a description of the trial. Ann Surg Oncol 2004;11S:208-10.

[6] Giuliano AE, Kirgan DM, Guenther JM, et al. Lymphatic mapping and sentinel lymphadenectomy for breast cancer. Ann Surg 1994;220(3):391-8 [discussion: 398-401].

[7] Albertini JJ, Lyman GH, Cox C, et al. Lymphatic mapping and sentinel node biopsy in the patient with breast cancer. JAMA 1996;276(22):1818-22.

[8] Veronesi U, Paganelli G, Galimberti V, et al. Sentinel-node biopsy to avoid axillary dissection in breast cancer with clinically negative lymph nodes. Lancet 1997;349(9069):1864-7.

[9] Canavese G, Gipponi M, Catturich A. Technical issues and pathologic implications of sentinel lymph node biopsy in early-stage breast cancer patients. J Surg Oncol 2001;77:81-7.

[10] McMasters KM, et al. Sentinel lymph node biopsy for breast cancer: a suitable alternative to routine axillary dissection in multi-institutional practice when optimal technique is used. J Clin Oncol 2000;18(13):2560-6. 
[11] Veronesi U, Paganelli G, Viale G, et al. Sentinel lymph node biopsy and axillary dissection in breast cancer: results in a large series. J Natl Cancer Inst 1999;91(4):368-73.

[12] Cox CE, Pendas S, Cox JM, et al. Guidelines for sentinel node biopsy and lymphatic mapping of patients with breast cancer. Ann Surg 1998;227(5):645-51 [discussion: 651-3].

[13] Bedrosian I, Reynolds C, Mick R, et al. Accuracy of sentinel lymph node biopsy in patients with large primary tumors. Cancer 2000;88:2540-5.

[14] Haigh PI, Hansen NM, Qi K, et al. Biopsy method and excision volume do not affect success rate of subsequent sentinel lymph node dissection in breast cancer. Ann Surg Oncol 2000; $7(1): 21-7$.

[15] Wong SL, Edwards MJ, Chao C, et al. The effect of prior breast biopsy method and concurrent definitive breast procedure on success and accuracy of sentinel lymph node biopsy. Ann Surg Oncol 2002;9(3):272-7.

[16] Krag D, Weaver D, Ashikaga T, et al. The sentinel node in breast cancer-a multicenter validation study. N Engl J Med 1998;339(14):941-6.

[17] O'Hea BJ, Hill AD, El-Shirbiny AM, et al. Sentinel lymph node biopsy in breast cancer: initial experience at Memorial Sloan-Kettering Cancer Center. J Am Coll Surg 1998;186(4):423-7.

[18] Guenther JM. Axillary dissection after unsuccessful sentinel lymphadenectomy for breast cancer. Am Surg 1999;65(10):991-4.

[19] Krag DN, et al. Surgical resection and radiolocalization of the sentinel lymph node in breast cancer using a gamma probe. Surg Oncol 1993;2(6):335-9.

[20] Newman LA. Lymphatic mapping and sentinel lymph node biopsy in breast cancer patients: a comprehensive review of variations in performance and technique. J Am Coll Surg 2004; 199(5):804-16.

[21] Lyew MA, Gamblin TC, Ayoub M. Systemic anaphylaxis associated with intramammary isosulfan blue injection used for sentinel node detection under general anesthesia. Anesthesiology 2000;93:1145-6.

[22] Kuerer HM, Wayne JD, Ross MI. Anaphylaxis during breast cancer lymphatic mapping. Surgery 2001;129:119-20.

[23] Montgomery LL, Thorne AC, Van Zee KJ, et al. Isosulfan blue dye reactions during sentinel lymph node mapping for breast cancer. Anesth Analg 2002;95:385-8.

[24] Simmons RM, Smith SM, Osborne MP. Methylene blue dye as an alternative to isosulfan blue dye for sentinel lymph node localization. Breast J 2001;7(3):181-3.

[25] Simmons R, Thevarajah S, Brennan M, et al. Methylene blue dye as an alternative to isosulfan blue dye for sentinel node localization. Ann Surg Oncol 2003;10(3):242-7.

[26] Blessing W, Stolier A, Teng S, et al. A comparison of methylene blue and lymphazurin in breast cancer sentinel node mapping. Am J Surg 2002;184:341-5.

[27] Kim T, Agboola O, Lyman G. Lymphatic mapping and sentinel lymph node sampling in breast cancer. Presented at the Proceedings of the American Society of Clinical Oncology 2002 Annual Symposium. 2002.

[28] Linehan DC, Hill AD, Tran KN, et al. Sentinel lymph node biopsy in breast cancer: unfiltered radioisotope is superior to filtered. J Am Coll Surg 1999;188(4):377-81.

[29] Borgstein PJ, Meijer S, Pijpers R. Intradermal blue dye to identify sentinel lymph node in breast cancer. Lancet 1997;349(9066):1668-9.

[30] Martin RC, Derossis AM, Fey J, et al. Intradermal isotope injection is superior to intramammary in sentinel node biopsy for breast cancer. Surgery 2001;130(3):432-8.

[31] Shen P, Glass EC, DiFronzo LA, et al. Dermal versus intraparenchymal lymphoscintigraphy of the breast. Ann Surg Oncol 2001;8(3):241-8.

[32] Klimberg VS, Rubio IT, Henry R, et al. Subareolar versus peri-tumoral injection for location of the sentinel lymph node. Ann Surg 1999;229(6):860-4 [discussion: 64-5].

[33] Beitsch PD, Clifford E, Whitworth P, et al. Improved lymphatic mapping technique for breast cancer. Breast J 2001;7(4):219-23.

[34] Kern KA. Sentinel lymph node mapping in breast cancer using subareolar injection of blue dye. J Am Coll Surg 1999;189:539-45. 
[35] Kern KA. Breast lymphatic mapping using subareolar injections of blue dye and radiocolloid: illustrated technique. J Am Coll Surg 2001;192:545-50.

[36] Tousimis E, Van Zee KJ, Fey JV, et al. The accuracy of sentinel lymph node biopsy in multicentric and multifocal invasive breast cancers. J Am Coll Surg 2003;197(4):529-35.

[37] Haigh PI, Hansen NM, Giuliano AE, et al. Factors affecting sentinel node localization during preoperative breast lymphoscintigraphy. J Nucl Med 2000;41(10):1682-8.

[38] McMasters KM, Wong SL, Tuttle TM, et al. Preoperative lymphoscintigraphy for breast cancer does not improve the ability to identify axillary sentinel lymph nodes. Ann Surg 2000;231(5):724-31.

[39] Borgstein PJ, Pijpers R, Comans EF, et al. Sentinel lymph node biopsy in breast cancer: guidelines and pitfalls of lymphoscintigraphy and gamma probe detection. J Am Coll Surg 1998;186(3):275-83.

[40] Winchester DJ, Sener SF, Winchester DP, et al. Sentinel lymphadenectomy for breast cancer: experience with 180 consecutive patients: efficacy of filtered technetium $99 \mathrm{~m}$ sulphur colloid with overnight migration time. J Am Coll Surg 1999;188(6):597-603.

[41] McCarter MD, Yeung H, Yeh S, et al. Localization of the sentinel node in breast cancer: identical results with same-day and day-before isotope injection. Ann Surg Oncol 2001; $8(8): 682-6$.

[42] Solorzano CC, Ross MI, Delpassand E, et al. Utility of breast sentinel lymph node biopsy using day-before-surgery injection of high-dose 99mTc-labeled sulfur colloid. Ann Surg Oncol 2001;8:821-7.

[43] Wilke LG, Giuliano A. Sentinel lymph node biopsy in patients with early-stage breast cancer: status of the national clinical trials. Surg Clin North Am 2003;83(4):901-10.

[44] American College of Surgeons Oncology Group. ACOSOG Z11 protocol. Available at: https://www.acosog.org/studies/organ_site/breast/archives/2004.jsp. Accessed April 3, 2007.

[45] Mamounas EP, Cohen L, Cohen A. Sentinel lymph node biopsy after neoadjuvant systemic therapy for breast cancer. Surg Clin North Am 2003;83(4):931-42.

[46] Breslin TM, Cohen L, Sahin A, et al. Sentinel lymph node biopsy is accurate after neoadjuvant chemotherapy for breast cancer. J Clin Oncol 2000;18(20):3480-6.

[47] Balch GC, Mithani SK, Richards KR, et al. Lymphatic mapping and sentinel lymphadenectomy after preoperative therapy for stage II and III breast cancer. Ann Surg Oncol 2003; 10(6):616-21. 